\title{
Differentiated Service of Streaming Media Playback Technology
}

\author{
CHENG Zi-ao ${ }^{1}$ MENG Bo ${ }^{1}$ WANG Dan-hua ${ }^{1}$ ZHAO Yue $^{1}$ \\ ${ }^{1}$ Informatization Center of Liaoning University
}

\begin{abstract}
With users increase in the personalized demand of streaming media service, to ensure the smooth transmission of streaming media and a reasonable reduction of resources has become a measure of the quality of streaming media services. According to distinguishing the type of services and taking the personalized improvement to segmented prefetching data and proxy server cache replacement which affects the streaming media technology, this paper proposes respectively SPDS algorithm and CRDS strategy. Simulation results show that these improvements can effectively reduce the proxy server load, reduce the waste of resources and network bandwidth consumption, while providing a better Qos.
\end{abstract}

Keywords: differentiated service; segmented prefetching; cache replacement

\section{Introduction}

With the growth of Mass inactive data and the integrity of real-time data, a complete set of the proxy server cache management strategies mainly include two aspects of data prefetch and cache replacement. From the aspect of time, by means of prefetching the segments of streaming media data, the user terminal can obtain streaming media information from the nearest proxy server; From the aspect of space, an efficient cache replacement strategy in proxy server, not only improve the effective use of physical resources, but also allow users to play smoothly streaming media file in the client feeling less than "caching data for waiting to playback" caused by "remote" server loading pressure. So, for these two aspects, this paper takes the personalized improvement to segmented prefetching data and proxy server cache replacement which affects the streaming media technology, and proposes respectively Segmentation Prefetching based on Differentiated service of Streaming Media (SPDS) and Cache Replacement based on Differentiated service of Streaming Media (CRDS) [1].

\section{Prefetching model of streaming media}

At present, many scholars have phased some models based on the probability of visiting, data mining, popularity and segmentation prefetching, etc. These several prefetching methods are only a single users' request as a separate streaming media data request. With diverse development of data services, Not only are the streaming data requested by the user operations different, but also their operational characteristics are different. This paper designs a cache replacement based on differentiated service of streaming media - SPDS which is based on the existing popularity prefetch model and combines the different characteristics and types of the streaming media service. 


\subsection{Streaming media service classifi- cation and characteristic}

In order to enable the terminal to playing on different service smoothly this paper proposes segmentation prefetching algorithm based on differentiated service of streaming media. According to the playback modes, the current streaming media services that are frequently-used can be divided into three types and combine the different segmentation strategies, as follow[1-2]:

Live service segmentation: Because of the requirement of the higher real-time, exponential segmentation prefetching algorithm (ESPA) is adopted which has high growth. As early as possible data are prefetched and cached in proxy server cache to ensure smooth playback by the users.

On-demand service segmentation: The real-time requirement of on-demand service is inferior to that of live service, which above download. To ensure a certain number of data blocks in the proxy server cache, so the twice sum of even number segmentation prefetching algorithm (TSENSPA) is adopted.

Download service segmentation: The uniform segmentation prefetching algorithm (USPA) is adopted, because its real-time is lower, which of data block is the least number, each is more complete in proxy server cache.

Streaming service processing platform will generate call detail records (CDR) [4].By extracting the part of the streaming data information, the SCDR information is generated - Similar CDR.

The source server or the proxy server according to the user request to distinguishing streaming media file type identification, and then in SCDR a file is identified(as Identification Number : Type Of Server)that as follows:0 is On-Demand service; 1 is Live service; 2 is Download service.

\subsection{Segmentation algorithm}

This paper adopts first a segmentation prefetching algorithm [1] based on the twice sum of even number. In accordance with the respective characteristics of streaming media services, combining with USPA and ESPA . The paper proposed Segmentation Prefetching based on Differentiated service of Streaming Media- SPDS, which is better to solve the contradiction of USPA and ESPA. The size of prefetching segmentation data-Ln should be combined with the type of service under certain condition of bandwidth and data. The proxy server will make full use of resources and improve the playback of real-time and continuous.

Case 1: When $\mathrm{Bs}>\mathrm{Bt}$, and $\Delta\left(\frac{B_{s}}{B_{t}}\right)<1$, $\mathrm{n}>0$,in this case, streaming media file which the proxy server receive are smaller than one that sent to the user terminal, and so user terminal playback speed, the proxy server using the classification of streaming media segments to prefetching strategy. Prefetching position $\mathrm{x}$ should satisfy the following formula:

$$
\begin{gathered}
\frac{L_{n}-x+L_{n+1}}{B_{s}} \geq \frac{L_{n+1}}{B_{t}} \\
L_{n}=\left[\alpha \cdot 2 n(n+1)+\beta \cdot 2^{n}+\gamma\right] M
\end{gathered}
$$

When the proxy server determines the type of streaming media, we can assign $[\alpha$, $\beta, \gamma]$ according to the identifier of SCDR type. As follows that: On-Demand service: $[\alpha, \beta, \gamma]=[1,0,0]$; Live service: $[\alpha$, $\beta, \gamma]=[0,1,0]$;Download service: $[\alpha, \beta$, $\gamma]=[0,0,1]$.

Case 2: when Bs $<\mathrm{Bt}$, streaming media file which the proxy server receive are larger than one that sent to the user terminal, as well as in case 1 , if the proxy server cache partial streaming data for a long period of time, there is no reasonable updated for streaming data. As there were insufficient cache resources of proxy server, both that and user terminal 
playback would cause jitter. In order to more fully using proxy server to cache resources, this paper will introduce a new cache replacement algorithm to solve this problem.

\section{Differentiated type of streaming media services and cache re- placement}

The thinking of traditional cache replacement policy is "by exchanges old". And the so-called "old" media objects may be that user perfetch the most content, which is replace the "new" streaming object to affect the user's playback.

In accordance with the abovedescribed improvement of prefetching algorithms, this paper combines the influential factor of streaming data playback with the cache technology of proxy server, to improve "cache replacement" for streaming media in proxy server. And that predict data from the popularity of streaming media data, transmission rate and the jitter of transmission rate. By means of which, the cache priority (utility value size) of streaming media data in the proxy server will be reset. This paper designs a cache replacement strategy based on differentiated service of streaming media-CRDS [1, 3].

\subsection{Parameter of CRDS strategy}

At present, the assessment model of the file popularity is mainly based on the Zipf distribution. According to the fact that the probability of i-th segment accessed follows a Zipf distribution $(\theta=0.271)$, it is used as a quota of the cached data priority by popularity as follows:

$$
T_{i}=\frac{\frac{1}{\sum_{i=1}^{n} i^{\theta-1}}}{i}=\frac{i^{\theta}}{\sum_{i=1}^{n} i^{\theta-1}}
$$

To using GM $(1,1)$ prediction model combining with historical statistical data (the average transfer rate of unit data segment- $\mathrm{R}_{\mathrm{ci}}$, that predict the transfer rate value- $R_{p i}$ of in the next period $i$-th segment data. The paper designs a trend factor of transmission rate through the comparison of the predicted values and the sample value- $Q_{i}$, in order to calculate the mean- $\mu$ of the trend factor of transmission rate in the range of sample time, to express the trend change of the data transmission rate for the next segment, and also design another one of the jitter level of transmission rate trend in the process- $\sigma^{2}$.

$$
\begin{gathered}
\mu=E(Q)=\bar{Q}=\frac{\sum_{k=i+1}^{j} \frac{R_{P_{i}}}{R_{C_{k}}}}{j-i} \\
\sigma^{2}=D(Q)=E\left\{[Q-E(Q)]^{2}\right\}
\end{gathered}
$$

\subsection{Utility function of CRDS strategy}

Each streaming data which have been cached or would be cached, it must make detailed statistical analysis on the three quotas. Seen by the utility function, utility value conferred by each data segment is proportional to the popularity and the transmission rate, and is inversely proportional to the jitter level of the transmission rate. The data requested in the proxy server are calculated its utility value which stored in the list of proxy server with the data segment information, in order to facilitate users proxy server storing and managing the cached streaming data when user requests that. So through three quotas, it could be more reasonable assessment of the priority level of cached streaming data segments in the proxy server, thereby reasonable using resources.

$$
\delta_{i}=\frac{T \cdot \mu}{\sigma^{2}}
$$

According to the characteristic about high real-time, in this paper it is defined 
that the live service cache must own the highest priority and the cache priority of the on-demand is lower than the live one, but higher than the download one. When it is directed by proxy server, the segments will be cached in the high cache. After all the live segments sent out to the end user terminal, the cache space occupied once would be released. If the cache space of live service has been full while the proxy server will cache the new live segments, the part cache of the ondemand service cache will be occupied and so on. When there are no spaces in the proxy server, which will use the CRDS strategy for the streaming media data segments and replace the space occupied by the lowest priority of the download service segments which do not contain the being accessed ones. After this, there are still no enough spaces, the segments being lower priority of the download service segments will released and so on until there are enough ones caching the live service segments. When the live service finishes playing, the spaces are released. The priority of the on-demand service is inferior to the one of live service. When the spaces of the on-demand are full while the proxy server will cache the new on-demand segments, the ones occupy the cache spaces of the download. The basic strategy is similar to the live one. The priority of the download service is the lowest, so when there are the new ones needing be cached and no enough spaces, the proxy server only replaces the lower utility value to satisfy that.

\section{Experiment}

Table 1. Proxy server testing parameters list [1-4]

\begin{tabular}{c|c}
\hline Parameter name & Parameter size \\
\hline $\begin{array}{c}\text { The size range of } \\
\text { streaming files }\end{array}$ & $100 \mathrm{MB}$ to $500 \mathrm{MB}$ \\
\hline $\begin{array}{c}\text { The number of } \\
\text { streaming media } \\
\text { files }\end{array}$ & 1000 \\
$\begin{array}{c}\text { The average interval } \\
\text { time of request }\end{array}$ & $10 \mathrm{~s}$ \\
\hline $\begin{array}{c}\text { The file popularity } \\
\text { The network delay } \\
\text { of proxy server and } \\
\text { user terminal }\end{array}$ & a Zipf distribution \\
$(\theta=0.271)$
\end{tabular}

The experiment shows that the segmentation prefetching and cache replacement strategy based on differentiated service has the advantage of the byte hit ratio in the sample time than the simplex one. When the proxy server cache occupied below $40 \%$, the byte hit ratio is basically consistent between them. When above $40 \%$, the SPDS-CRDS algorithm exhibits slightly advantage. 

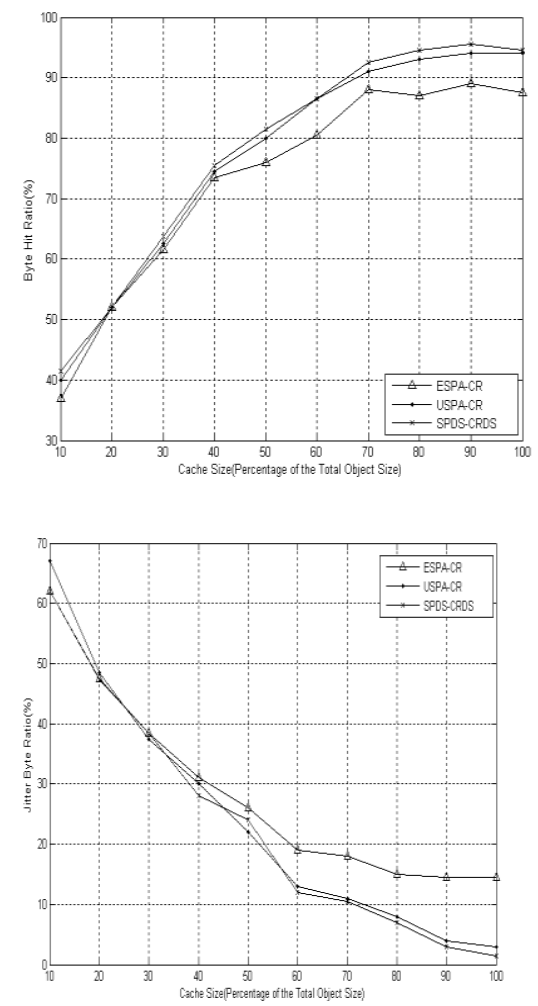

Figure 1 . The byte hit ratio and jitter ratio of the three algorithms

\section{Conclusions}

According to the user needing more streaming media service types and the common streaming media playback modes, these are divided into three cate- gories and experimented. But the new service are constantly emerging, in order to better meet the needs of the users and the extensive development, it is still needed making the necessary adjustments to some parameters impacting on cache replacement.

In short, I believe that with the development computer network and the investment and construction by ISP, these problems will be solved.

\section{References}

[1] Cheng Zi-ao. Research and Design of Differentiated Service of Streaming Media Play Technology [D], Liaoning University, 2011.

[2] DAI Zhong, YANG Ge, LIAO Jianxin, ZHU Xiao-min, and HUANG Hai."Active prefetching algorithm for streaming media based on natural number segmentation," Journal on Communications [J], vol. 29 No.3 March 2008:98-103

[3] YANG Chuandong,YU Zhenwei,WANG Xinggang,ZHANG Huanyuan.Proxy Cache Replacement Algorithm Based on Popularity Prediction of Streaming Media File of Streaming Media File.Computer Engineering $[\mathrm{J}]$.vol.33 No.7April

[4] General Technical Requirements Of Mobile Streaming Service in Digital Cellular Mobile Telecommunication Network, Ministry Of Information Industry Of the People' s Republic Of China, YD/T 1489-2006. 